\title{
Marat Arakelian: Life and Scientific Activity
}

\author{
A. M. Mickaelian* \\ NAS RA Byurakan Astrophysical Observatory (BAO), Byurakan 0213, Aragatzotn Province, Armenia
}

\begin{abstract}
We review the life and scientific activity of one of the outstanding Armenian astronomers Marat Arakelian (1929-1983). Arakelian was one of the prominent Byurakan astronomers, the author of famous Arakelian galaxies, which at present are target for many-sided studies with ground-based and space telescopes. Arakelian is known as a distinguished specialist in the theoretical astrophysics and extragalactic astronomy.
\end{abstract}

Keywords: active galaxies, AGN, Starburst Galaxies, quasars, Seyfert galaxies, LINERs

\section{Introduction}

Prof. Arakelian is one of the prominent Byurakan astronomers, the author of famous Arakelian galaxies, which at present are target for many-sided studies with ground-based and space telescopes. Arakelian is known as a distinguished specialist in the theoretical astrophysics and extragalactic astronomy.

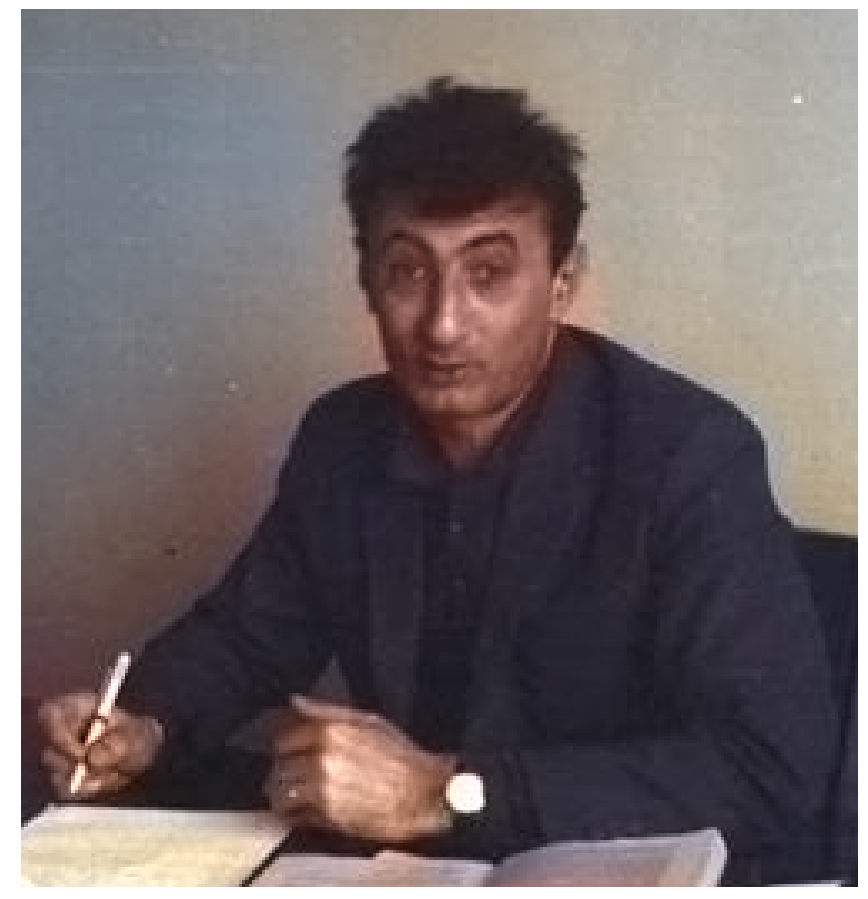

\section{Life and Activities}

Marat Arsen Arakelian was born on January 15, 1929, in Goris, Armenia, USSR. He studied at the Physical-Mathematical Department of the Yerevan State University (YSU) and graduated from

*aregmick@yahoo.com, Corresponding author 
it in 1951, among the first students specialized on Astrophysics. He was directed to the Byurakan Astrophysical Observatory (BAO), where he worked first as assistant astronomer, and later as junior research associate. Soon he became a postgraduate student at the Leningrad State University (LSU, presently, St. Petersburg), finished the studies in 1955, and in 1956 successfully passed his Ph.D. thesis "Spectrophotometric investigation of Algol" under the supervision of Prof. O.A. Melnikov at the LSU.

In 1957-1959 Arakelian combines his work with senior teacher position at the Department of Astrophysics of the YSU. From 1960 to 1966 he was a junior researcher and lectured at the LSU. He was awarded the title of Associate Professor. Later on, since 1966 Arakelian again works at BAO and combines his research with a position of a lecturer at the Department of Astrophysics of the YSU. In 1967, he became a senior researcher at BAO and headed an important direction in the extragalactic astronomy.

Since 1967, Arakelian completely devoted himself to the research work and during a short period performed a fantastic productivity for those times, publishing 62 papers in 1968-1983, and giving important scientific results in almost each of these works!

In 1977, Arakelian defended his second Doctoral Degree thesis "Spectral observations and statistics of galaxies with active nuclei" at the Moscow State University (MSU) and became a Doctor of Sciences.

Arakelian combined his scientific work with pedagogical one as well. In 1982 he was elected the Chair of the Department of Astrophysics and Theoretical Physics of the Armenian State Pedagogical Institute after Kh. Abovian. Together with L. V. Mirzoyan, A. T. Kalloghlian, and H. M. Tovmassian, he was the co-author of the textbook "Astronomy" for secondary schools (three editions in 1970, 1971, and 1973, in Armenian). Arakelian has written an extended review on Clusters of galaxies in the book "Problems of extragalactic astronomy" (1981).

Arakelian has published about 80 papers in various astrophysical journals, including such prestigious journals, as Nature, Astronomical Journal, and Astrophysical Journal (one of the rare Byurakan astronomers to publish a paper in Nature), as well as in proceedings of several international conferences. He was the editor of the two proceedings books of the Byurakan meetings: "The Non-Stable Stars" (1956) and the IAU Symp. \#29 "Non-Stable Phenomena in Galaxies" (1966).

Since 1973, Arakelian was a member of the International Astronomical Union (IAU), Commission \#28 "Galaxies".

M. A. Arakelian passed away very early, at the age of 54 years on January 20, 1983, in Moscow, when he was at the period of prosperity of his scientific activity.

\section{Scientific Results and Achievements}

We give here the most important results and achievements by Marat Arakelian.

1968, Study of the luminosity function and the stellar space density in the Solar neighborhood. The results were published in Astrophysics.

1968-1969, Study of the luminosity evolution of quasars based on the evolutionary effects associated with them. The results were published in Astrophysics (Астрофизика, 2 papers in 1969-1970), in Soviet Astronomical Circular (Астрономический ииркуляр), and a summary of these works was published in the prestigious journal Nature in 1970 (vol. 225, p. 358-359).

1969-1970, Statistical study of flare stars in the solar vicinity. The results were published in Commumications of the Konkoly Observatory, Communications of BAO, and were reported at the conference "Non-periodic phenomena in variable stars" in 1969.

1970, Derivation of the luminosity function of field galaxies (together with A. T. Kalloghlian). The results were published in Soviet Astronomy (Астрономический журнал).

1970-1971, The proof of the extragalactic origin of quasars. The results were published in Astrophysics and Becтник AH CCCP (Bulletin of the USSR Acad. Sci.). 
1970-1973, Spectroscopic observations and studies of a few hundred Markarian galaxies and discovery of more than 40 new Seyferts among them (together with Russian astronomers E.A. Dibai and V.F. Esipov). The results were published in series of 8 papers in Astrophysics and 5 papers in the Soviet Astronomical Circular.

1972-1974, Analysis of the surface brightness of emission-line galaxies (including Seyfert and Markarian ones) and development of method for revealing galaxies with high surface brightness. The results were published in 3 papers in Astrophysics.

1973, Suggestion of a new method for definition of space density of extragalactic objects and estimation of the mean density of matter in the Metagalaxy. The results were published in Astrophysics.

1975, Compilation and publication of the catalogue of "Galaxies of high surface brightness" (named Arakelian galaxies, Akn), a list of 621 objects with surface brightness at least 22.0 magnitudes from an area of 1 sq. arcsec. The sample contained $4 \%$ of all galaxies in an area of with $\delta>-3^{\circ}$ and $|\mathrm{b}|>20^{\circ}$. Arakelian catalog became a source for many new AGN (Communications of BAO, No. 47, p. 3-42, 1975).
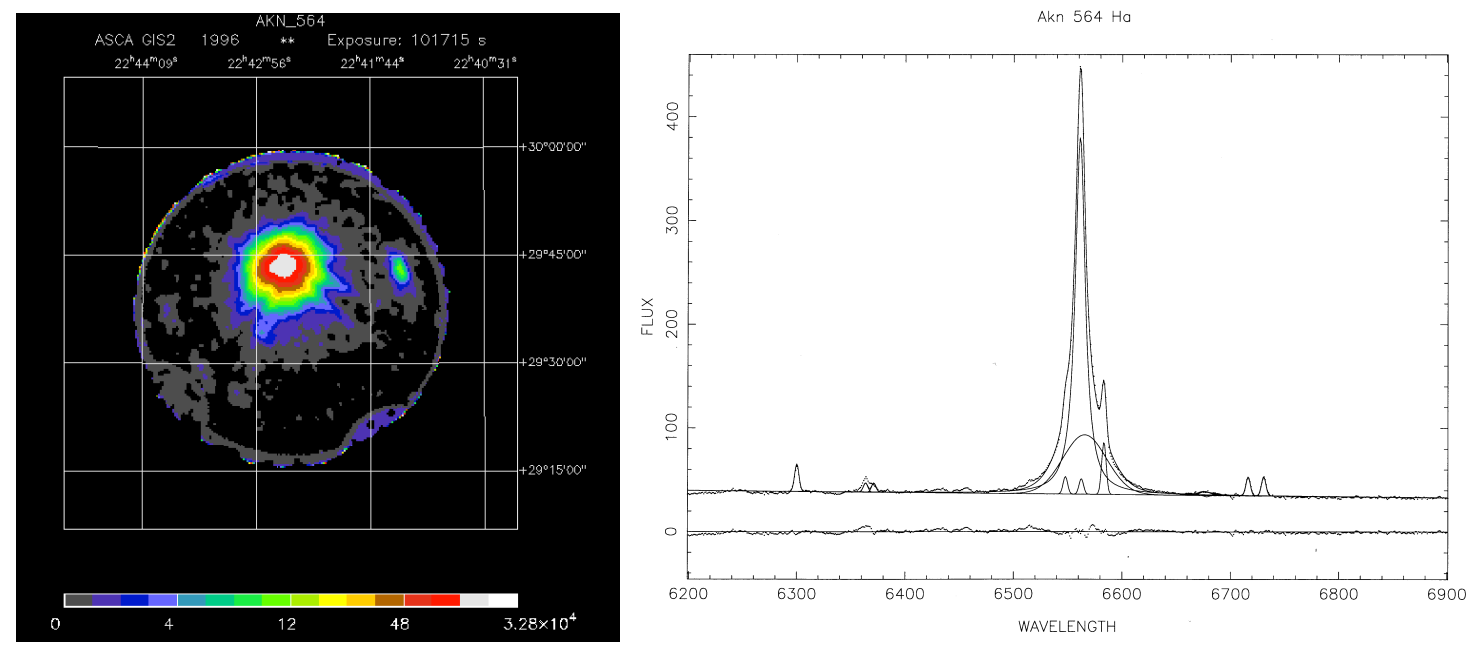

1975, Derivations of the luminosity function and space density of galaxies with UV continuum (Markarian galaxies). The results were published in Soviet Astronomy.

1975-1976, Spectroscopic observations and studies of Arakelian galaxies (galaxies with high surface brightness) and discovery of new Seyferts among them (together with E. A. Dibai and V. F. Esipov). The results were published in a series of 4 papers in Astrophysics, a paper in the Soviet Astronomical Circular, and were reported in the meeting "Stars and galaxies from observational points of view" in 1975.

1976-1977, Study of the dependence of the emission-line intensities of Markarian and Seyfert galaxies on their color index. The results were published in 2 papers in Astrophysics.

1977, Study of the distribution of the mean surface brightness of galaxies in the Coma cluster. The results were published in Astrophysics.

1977-1980, Study of the relation between the mean surface brightness and radio emission of galaxies, including Seyfert galaxies (together with R. A. Kandalyan). The results were published in 2 papers in Astrophysics.

1981, Study of the estimations of the kinetic energies of clusters of galaxies; the extent to which a kinetic energy estimate would be affected by a possible mass dependence of the velocity dispersion of galaxies in clusters was considered. It was concluded that in some cases the kinetic energy might be underestimated (together with A. G. Kritsuk). The results were published in Astrophysics. 
1980-1981, Radio (6cm) observations of Arakelian galaxies and publication of their accurate positions (together with USA astronomers G. Kojoian, D. F. Dickinson, R. Elliott, M. D. Bicay). The results were published in the Astronomical Journal.

1981-1982, Comparative study and statistics of the surface brightness and morphological types of isolated and double galaxies (together with A. P. Mahtessian). The results were published in 2 papers in Astrophysics.

1981-1982, Study of Seyfert galaxies in clusters and Seyfert properties of the cluster galaxies (together with V. Yu. Terebizh). The results were published in the Soviet Astronomical Circular and Soviet Astronomy Letters (Писъма в Астрономический журнал).

1983, Proposing a method for construction of the luminosity function of the components of double galaxies on the basis of an arbitrary sample of pairs of galaxies. The results were applied to the data of Karachentsev's Catalog of Isolated Pairs. The presence of a correlation between the absolute magnitudes of the components of pairs was confirmed. The results were published in Astrophysics.

1986, A method for the determination of the bivariate luminosity function utilizing an incomplete sample with an application to Seyfert galaxies. It was shown that the results of incomplete radio and X-ray surveys could be used for the determination of the bivariate luminosity functions, having both the distribution of luminosities of objects in the detected subsample and the distribution of apparent magnitudes in the surveyed sample. A paper was published in Astrophysical Journal after Arakelian's death.

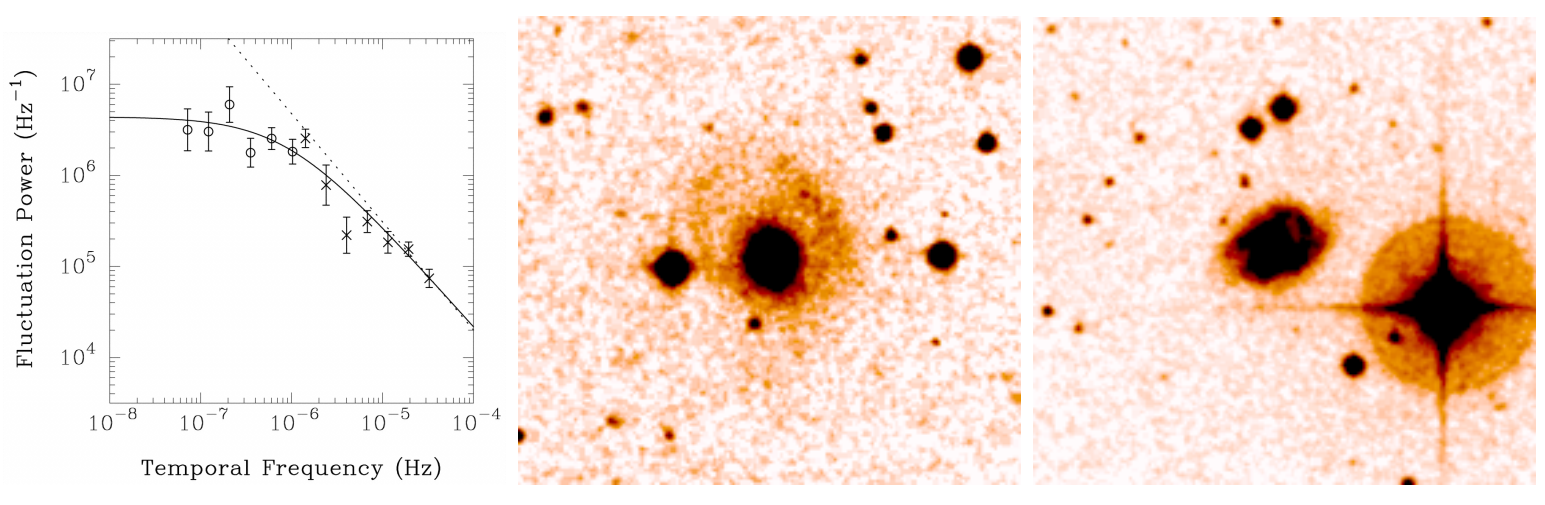

\section{Chronology of Marat Arakelian's life and activity}

1929

1946-1951

1951

1953-1955

1956

1957-1959

1960-1966

1965:

1966-1983

1967

1968-1969

1970-1971

1970-1973
Marat Arsen Arakelian was born on January 15 in Goris, Armenia, USSR studies at the Phys.-Math. Department of the Yerevan State University (YSU) starting working at the Byurakan Astrophysical Observatory (BAO) (first as assistant astronomer, and later as junior research associate) postgraduate student at the Leningrad State University (LSU)

Ph.D. thesis "Spectrophotometric investigation of Algol" (supervisor: Prof. O. A. Melnikov)

senior teacher at the Department of Astrophysics of the YSU junior researcher and lecturer at the LSU

Associate Professor at the LSU

work at BAO and lecturer at the Department of Astrophysics of the YSU

senior researcher at $\mathrm{BAO}$, head of a group

study of the luminosity evolution of quasars, a paper in Nature in 1970

the proof of the extragalactic origin of quasars

spectroscopic observations and studies of a few hundred Markarian galaxies (together

with E. A. Dibai and V. F. Esipov) 
1973 suggestion of a new method for definition of space density of extragalactic objects and estimation of the mean density of matter in the Metagalaxy

1973 member of the International Astronomical Union (IAU), Commission \#28 "Galaxies"

1975 compilation and publication of the catalogue of "Galaxies of high surface brightness" (named Arakelian galaxies, Akn), a list of 621 objects

1975-1976 spectroscopic observations and studies of Arakelian galaxies (together with E. A. Dibai and V. F. Esipov)

1977 Doctoral thesis "Spectral observations and statistics of galaxies with active nuclei" at the Moscow State University (MSU)

1982 Chair of the Department of Astrophysics and Theoretical Physics of the Armenian State Pedagogical Institute after Kh. Abovian

1983 proposing a method for construction of the luminosity function of the components of double galaxies on the basis of an arbitrary sample of pairs of galaxies

1983

M. A. Arakelian passed away on January 20 in Moscow

\section{Summary of publications}

Here we give the summary of Marat Arakelian's all publications by journals or other editions, including the numbers and years. In total, there should be 76 publications, however some are missing in the list. Most important journals and editions are in bold.

\begin{tabular}{|c|c|c|}
\hline Journal / edition & Number of publications & Years \\
\hline Nature & 2 & 1968,1970 \\
\hline Astronomical Journal (AJ) & 1 & 1981 \\
\hline Astrophysical Journal (ApJ) & 1 & 1986 \\
\hline Astronomy Reports (Астрон. Ж..) & 4 & $1959-1975$ \\
\hline Astronomy Letters (ПАЖK) & 2 & 1982 \\
\hline $\begin{array}{l}\text { Reports USSR Acad. Sci., Series: Physics (ДАН } \\
\text { CCСР, серия Физика) }\end{array}$ & 1 & 1968 \\
\hline Astrophysics (Астрофизика) & 31 & 1968-1983 \\
\hline Bull. USSR Acad. Sci. (Вестник AH CCCP) & 2 & 1969,1971 \\
\hline Communications of $\mathrm{BAO}$ & 3 & 1970-1975 \\
\hline Communications of Konkoly Obs. & 1 & 1969 \\
\hline Astronomical Circular (Астрон. Циркуляр) & 8 & $1970-1981$ \\
\hline BAAS & 2 & $1980-1981$ \\
\hline Proceedings of meetings & 3 & $1969,1976,1978$ \\
\hline Theses (PhD, Doctoral) & 2 & 1956,1977 \\
\hline Astronomy textbooks (in Armenian) & 3 & $1970,1971,1973$ \\
\hline Clusters of Galaxies (in Book) & 1 & 1981 \\
\hline Editing of Proc. of Byurakan Symposia & 2 & 1956,1966 \\
\hline Total & 69 & 1956-1986 \\
\hline
\end{tabular}

\section{Summary}

Arakelian has compiled and published the catalog of 621 high-surface-brightness galaxies, has proved the extragalactic origin of quasars, has determined the space density of extragalactic objects, has proposed several methods for study of properties of extragalactic objects, has made a comparative analysis of properties of different types of galaxies, and with his Russian colleagues spectroscopically has observed and studied some 800 faint galaxies and quasars. Arakelian galaxies have been observed in many observatories in the USA, UK, USSR, and elsewhere in optical wavelengths, radio and X-rays. Especially interesting objects are Akn 120 and Akn 564 (both are strongly variable AGN in X-ray and optical wavelengths; Akn 564 is a Narrow-Line Seyfert 1 galaxy that strongly changes the intensities of its emission lines). 
Many Arakelian galaxies are AGN or Starbursts and entered the Véron-Cetty \& Véron (2010) catalogue, as well as many are X-ray sources and have been detected by ROSAT (Voges et al. 1999, 2000), and many are radio sources and have been detected by NVSS (Condon et al. 1998), FIRST (Helfand et al. 2015) and other surveys. Many spectra of Arakelian galaxies have been observed by SDSS (Aguado et al. 2019) and are subject of appropriate classification for activity types.

\section{References}

Aguado D. S., Romina A., Andrés A., et al. 2019, ApJS, 240, id. 23

Condon J., Cotton W., Greisen E., et al. 1998, AJ, 115, 1693

Helfand D. J., White R., Becker R. H., 2015, ApJ, 801, id. 26

Véron-Cetty M. P., Véron P., 2010, A\&A, 518, A10

Voges W., Aschenbach B., Boller T., et al. 1999, A\&A, 349, 389

Voges W., Aschenbach B., Boller T., et al. 2000, MPE, Garching. The ROSAT Faint Source Catalogue

\section{List of research papers by Marat Arakelian (1956-1986)}

Arakelian, M. A. 1956, Spectrophotometric investigation of Algol, PhD Thesis

Dolidze, M. V.; Arakelyan, M. A. 1959, The T-Association Near $\rho$ Ophiuchi, SvA 3, 434 (AZh $36,444)$

Arakelian, M. A. 1961, Application of the Probability Method to the Determination of Absorption - Line Profiles, SvA 4, 945 (AZh 37, 1012)

Arakelian, M. A. 1968, Determining the Luminosity Function and Star Density in the Neighborhood of the Sun, PhD Thesis 13, 190

Arakelian, M. A. 1968, Luminosity function and stellar density in the neighborhood of the Sun, Ap 4, 256 (Afz 4, 617)

Arakelian, M. A. 1968, Distances, Space Distribution and Luminosity Function of Quasi-stellar Objects, Nature 219, 595

Arakelian, M. A. 1969, Curve of growth for an expanding atmosphere, Ap 5, 32 (Afz 5, 75)

Arakelian, M. A. 1969, Evolutionary effects associated with quasi-stellar radio sources. I, Ap 5, 226 (Afz 5, 461)

Arakelian, M. A. 1969, Nature of evolutionary effects connected with quasi-stellar radio sources. II, Ap 5, 308 (Afz 5, 603)

Arakelian, M. A.; Kalloglyan, A. T. 1970, The Luminosity Function of Field Galaxies, SvA 13, 953 (AZh 46, 1215, 1969)

Arakelian, M. A.; Ivanov, V. V.; Tovmasyan, G. M. 1969, Physics of stars, nebulae and galaxies (Symposium at Byurakan, 1968 Sep 16-20), VeSSR 3, 125

Arakelian, M. A.; Dibai, E. A.; Esipov, V. F. 1970, Spectra of Markarian galaxies. I, Ap 6, 14 (Afz 6, 39)

Arakelian, M. A.; Dibai, E. A.; Esipov, V. F.; Markaryan, B. E. 1970, Spectra of Markarian galaxies. II, Ap 6, 189 (Afz 6, 357) 
Arakelian, M. A. 1970, The spatial distribution and luminosity function for quasistellar radio sources, Ap 6, 291 (Afz 6, 531)

Arakelian, M. A. 1970, Evolution of the luminosity of quasars, ATsir 552, 1

Arakelian, M. A.; Dibaj, Eh. A.; Esipov, V. F.; Markarian, B. E. 1970, New Seyfert-type objects in the second Markarian's list, ATsir 568, 1

Arakelian, M. A.; Dibaj, Eh. A.; Esipov, V. F.; Markarian, B. E. 1970, New Seyfert-type objects in the third Markarian's list, ATsir 568, 2

Arakelian, M. A. 1970, Evidence of Radio Luminosity Evolution of Quasi-stellar Radio Sources, Nature 225, 358

Arakelian, M. A. 1970, On the statistics of flare stars in the solar vicinity, SoByu 41, 56

Arakelian, M. A. 1971, On the distances of quasi-stellar radio sources, VeSSR 11, 26

Arakelian, M. A.; Dibai, E. A.; Esipov, V. F.; Markaryan, B. E. 1971, Spectra of Markarian galaxies. III, Ap 7, 102 (Afz 7, 177)

Arakelian, M. A. 1971, Quasistellar radio sources and optical quasistellar objects, Ap 7, 269 (Afz $7,457)$

Arakelian, M. A.; Dibai, E. A.; Esipov, V. F. 1972, Spectra of Markarian galaxies. IV, Ap 8, 17 (Afz 8, 33)

Arakelian, M. A.; Dibai, E. A.; Esipov, V. F. 1972, Spectra of Markarian galaxies. V, Ap 8, 106 (Afz 8, 177)

Arakelian, M. A.; Dibai, E. A.; Esipov, V. F. 1972, Spectra of Markarian galaxies. VI, Ap 8, 197 (Afz 8, 329)

Arakelian, M. A. 1972, Estimates of the surface brightness of selected Markarian galaxies. I, Afz 8,473

Arakelian, M. A. 1972, Relationship between the gradient of the surface brightness and other characteristics of Seyfert galaxies, Ap 8, 368 (Afz 8, 624)

Arakelian, M. A.; Dibaj, Eh. A.; Esipov, V. F. 1972, On the spectrum of the Markarian galaxy 388, ATsir 717,7

Arakelian, M. A.; Dibaj, Eh. A.; Esipov, V. F. 1972, New Seyfert-type objects from the IIIrd and IVth lists of galaxies with UV-continuum, ATsir 722, 3

Arakelian, M. A. 1973, Estimate of the mean density of matter in the Metagalaxy, Ap 9, 84 (Afz $9,151)$

Arakelian, M. A.; Dibai, E. A.; Esipov, V. F. 1973, Spectra of Markaryan galaxies. VII, Ap 9, $180($ Afz 9, 319)

Arakelian, M. A.; Dibai, E. A.; Esipov, V. F. 1973, Spectra of Markaryan galaxies. VIII, Ap 9, $183($ Afz 9, 325)

Arakelian, M. A. 1974, Surface brightness of galaxies with emission lines, Ap 10, 321 (Afz 10, 507)

Arakelian, M. A. 1975, Luminosity function and space density of galaxies with an ultraviolet continuum, SvA 18, 432 (AZh 51, 730, 1974)

Arakelian, M. A. 1975, Galaxies of high surface brightness, SoByu 47, 3 
Arakelian, M. A. 1975, Classification of the Central Regions of 711 Galaxies, SoByu 47, 43

Arakelian, M. A.; Dibai, E. A.; Esipov, V. F. 1975, Spectral observations of galaxies with high surface brightness. I, Ap 11, 8 (Afz 11, 15)

Arakelian, M. A.; Dibai, E. A.; Esipov, V. F. 1975, Spectral observations of galaxies with high surface brightness. II, Ap 11, 254 (Afz 11, 377)

Arakelyan, M. A.; Dibai, E. A.; Esipov, V. F. 1976, Spectral observations of high-surfacebrightness galaxies. III, Ap 12, 122 (Afz 12, 195)

Arakelian, M. A. 1976, Dependence of the emission-line intensity of Markaryan galaxies on color index, Ap 12, 366 (Afz 12, 559)

Arakelian, M. A.; Dibai, E. A.; Esipov, V. F. 1976, Spectral observations of high-surfacebrightness galaxies. IV, Ap 12, 456 (Afz 12, 683)

Arakelian, M. A.; Dibaj, Eh. A.; Esipov, V. F. 1976, New Seyfert-type objects, ATsir 914, 7

Arakelian, M. A. 1976, Some results of spectral observations of galaxies of high surface brightness, sgov meet 297

Arakelian, M. A. 1977, Mean surface brightness and radio emission of galaxies, Ap 13, 130 (Afz $13,245)$

Arakelian, M. A. 1977, Dependence of the emission line intensities of Seyfert galaxies on the color index, Ap 13, 241 (Afz 13, 427)

Arakelian, M. A. 1977, The distribution of the mean surface brightnesses of galaxies in the Coma cluster, Ap 13, 386 (Afz 13, 651)

Arakelian, M. A. 1978, Markarian Galaxies in the Vicinity of the Coma Cluster, IAU S79, 274

Kojoian, G.; Dickinson, D. F.; Arakelian, M. A. 1980, Centimeter Wavelength Observations of Arakelian Galaxies, BAAS 12, 822

Arakelian, M. A.; Kandalyan, R. A. 1980, Mean Surface Brightnesses and Radio Emission of Seyfert Galaxies, Ap 16, 383 (Afz 16, 663)

Arakelian, M. A.; Magtesyan, A. P. 1981, Mean Surface Brightnesses of Isolated and Double Galaxies, Ap 17, 28 (Afz 17, 53)

Arakelian, M. A.; Terebizh, V. Y. 1981, Seyfert Galaxies in the Abell Cluster, ATsir 1188, 1

Arakelian, M. A. 1981, III. Clusters of galaxies, pea conf 83

Kojoian, G.; Dickinson, D.; Arakelian, M. A.; Bicay, M. D. 1981, Six-Centimeter Survey of Arakelian Galaxies, BAAS 13, 522

Kojoian, G.; Elliott, R.; Bicay, M. D.; Arakelian, M. A. 1981, Accurate optical positions of Arakelian galaxies, AJ 86, 820

Arakelian, M. A.; Kritsuk, A. G. 1981, On estimating the kinetic energies of clusters of galaxies, Ap 17, 384 (Afz 17, 709)

Arakelian, M. A.; Terebizh, V. Iu. 1982, Cluster galaxies with Seyfert properties, SvAL 8, 72

Arakelian, M. A.; Magtesyan, A. P. 1982, Spectrophotometry of Four Galaxies of High Surface Brightness, SvAL 8, 181 (PAZh 8, 334)

Arakelian, M. A. 1983, Statistics of morphological types of isolated galaxies and components of pairs, Ap 19, 375 (Afz 19, 665) 
Arakelian, M. A. 1983, Distribution of apparent magnitudes and luminosities of double galaxies, Ap 19, 379 (Afz 19, 673)

Arakelian, M. A. 1986, A Proposed Method for the Determination of the Bivariate Luminosity Function Utilizing an Incomplete Sample with an Application to Seyfert Galaxies, ApJ 301, 92 